\title{
Baryon form factors in the chiral quark-soliton model
}

\author{
A. Silva ${ }^{\mathrm{a}}$, D. Urbano ${ }^{\mathrm{b}}$ and K. Goeke ${ }^{\mathrm{c}}$
}

${ }^{a}$ Centro de Física Computacional e Departamento de Física, Universidade de Coimbra, Rua Larga, P-3004-516 Coimbra, Portugal

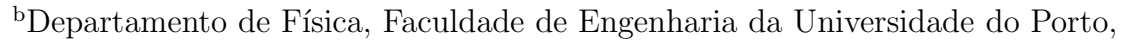

R. Roberto Frias, s/n, P-4200-465 Porto, Portugal

${ }^{\mathrm{c} I n s t i t u t ~ f u ̈ r}$ Theoretische Physik II, Ruhr-Universität Bochum, D-44780 Bochum, Germany

We present updated results for the electromagnetic form factors of the baryon octet and the axial form factors of the nucleon in the chiral quark-soliton model (CQSM).

\section{INTRODUCTION}

Form factors are among the most useful quantities in describing the extended structure of baryons. They have been therefore the subject of numerous theoretical and experimental investigations, still deserving a lot of attention at present, as reviewed in [1].

The chiral quark-soliton model (CQSM) has been successfully applied to the description of many baryon observables since it was first extended to flavour SU(3) in [2]. Reviews on the theoretical foundations of the model and on the framework which allows to compute physical observables may be found in [3] and [4], respectively. The CQSM is based on the $\mathrm{SU}(2)$ Lagrangian ( $M$ is the constituent quark mass, taken as a constant)

$\mathcal{L}=\bar{\psi}(x)\left(i \not \partial-m-M U^{\gamma^{5}}\right) \psi(x), \quad M U^{\gamma^{5}}=M e^{i \gamma^{5} \vec{\pi} \cdot \vec{\tau}}=\sigma+i \gamma^{5} \vec{\pi} \cdot \vec{\tau}$,

supplemented with the proper-time regularization. The parameters of the model are the regularization cut-off and the current quark masses and they are fixed in the meson sector. $M$ is the only free parameter, set to $M=420 \mathrm{MeV}$ for all form factors.

While the theoretical most important features of the model are chiral symmetry and spontaneous chiral symmetry breaking, the most important feature of the framework to calculate baryon observables is that of large- $N_{c}$, which justifies the semiclassical collective quantization of the rotational zero modes of the mean field $U_{c}(\mathbf{x})$. The collective coordinates are identified with the orientation $(A)$ of the soliton in flavour space and the quantization proceeds by using rotational motion constructed from the rotational zero modes, underlying the ansatz $U(\mathbf{x}) \rightarrow A(t) U_{c}(\mathbf{x}) A^{\dagger}(t)$. Observables in this work are calculated to first order both in the angular velocity $\Omega^{\alpha}$, from $A^{\dagger} \dot{A}(t)=i \Omega_{E}^{\alpha} \lambda^{\alpha} / 2$, and in the

\footnotetext{
*Acknowledges support from the grant PRAXIS XXI/BD/15681/98 and the Centro de Física
} Computacional da Universidade de Coimbra 

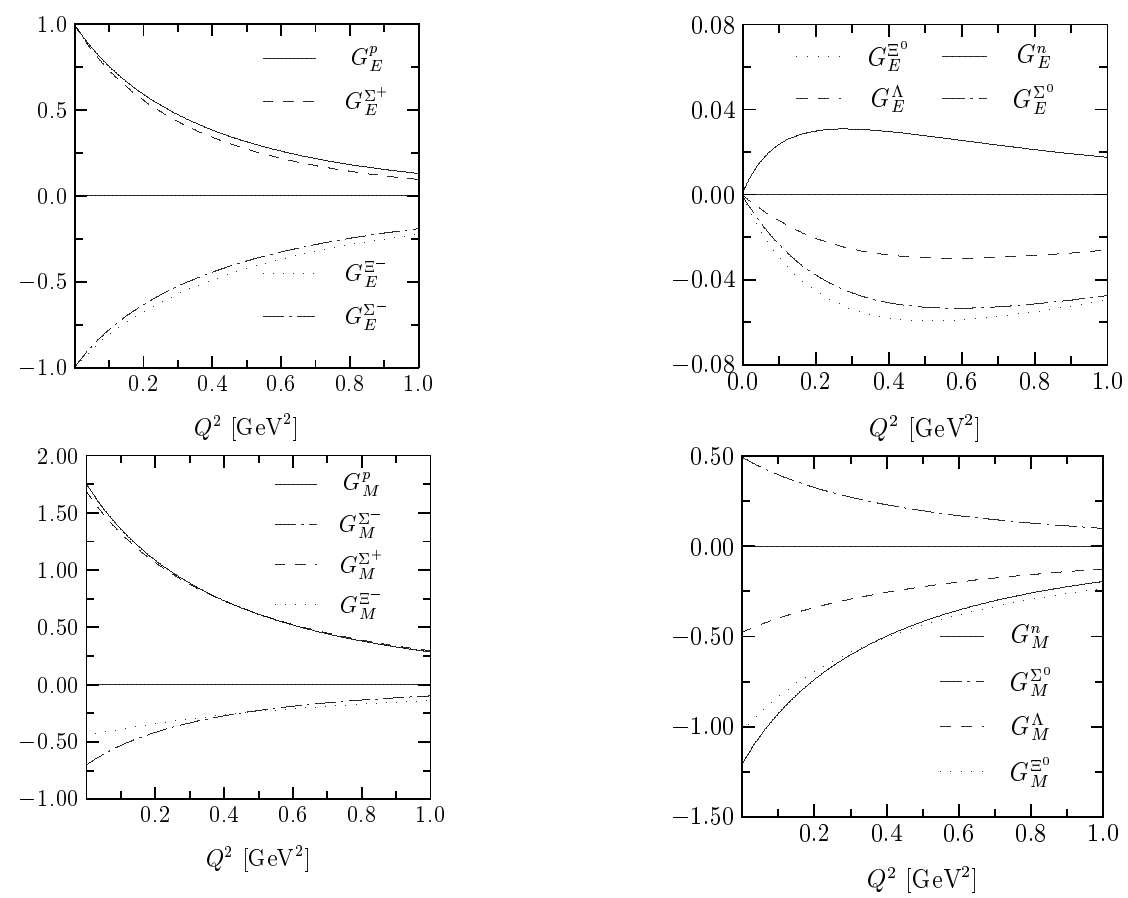

Figure 1. Electric (upper figures) and magnetic (lower) form factors for the charged (left) and neutral (right) octet baryons.

strange quark mass. The collective Hamiltonian contains a symmetry conserving term, treated exactly, and a symmetry breaking term, treated pertubatively. The extension of the formalism to flavour SU(3) rests mainly in embedding of the $\mathrm{SU}(2)$ mean field into a $\mathrm{SU}(3)$ matrix, i.e. $U=\exp \left(i P(r) \sum_{i=a}^{8} n^{a} \lambda^{a}\right) \rightarrow \operatorname{diag}\left(\exp \left[i P_{c}(r) \sum_{i=1}^{3} n^{i} \tau^{i}\right], 0\right)$.

\section{ELECTROMAGNETIC AND AXIAL FORM FACTORS}

The novelties in the present work regarding the electromagnetic form factors, shown in Fig. 1, consist in the use of the symmetry conserving quantization of [5] and the phenomenological inclusion of asymptotic meson effects. The new quantization prescription is necessary in order to identify and consistently remove spurious terms from the quantization procedure, which affected previous results [6]. The meson asymptotic effects are taken into account by using, for a given baryon $B$, the ansatz

$G_{E, M}^{B}\left(Q^{2}\right)=G_{E, M}^{u B(\pi)}\left(Q^{2}\right)+G_{E, M}^{d B(\pi)}\left(Q^{2}\right)+G_{E, M}^{s B(K)}\left(Q^{2}\right)$,

which is based on a flavor decomposition of the form factors, made possible by computing the form factors for the three octet currents $V^{0}, V^{3}$ and $V^{8}$. In this ansatz $\pi$ and $\mathrm{K}$ 
Table 1

Electric and magnetic radii $\left(\mathrm{fm}^{2}\right)$ and magnetic moments (n.m.) of the baryon octet.

\begin{tabular}{lrrrrrr}
\hline Baryon & $\left\langle r^{2}\right\rangle_{E}$ & \multicolumn{1}{c}{ Exp. } & $\left\langle r^{2}\right\rangle_{M}$ & Exp. & $\mu$ & Exp. \\
\hline$p$ & 0.728 & $0.729 \pm 0.024$ & 0.649 & $0.699 \pm 0.018$ & 1.759 & 2.793 \\
$n$ & -0.097 & $-0.113 \pm 0.007$ & 0.677 & $0.776 \pm 0.020$ & -1.210 & -1.913 \\
$\Lambda$ & 0.039 & & 0.457 & & -0.478 & -0.613 \\
$\Sigma^{-}$ & 0.662 & $\{0.61 \pm 0.21$ \\
$\Sigma^{0}$ & 0.075 & & 0.718 & & -0.702 & -1.16 \\
$\Sigma^{+}$ & 0.811 & & 0.550 & & 0.495 & \\
$\Xi^{-}$ & 0.546 & & 0.619 & & 1.692 & 2.458 \\
$\Xi^{0}$ & 0.102 & & 0.318 & & -0.444 & -0.651 \\
\hline
\end{tabular}

The experimental values for the $\Sigma^{-}$radius are from [7] and [8].

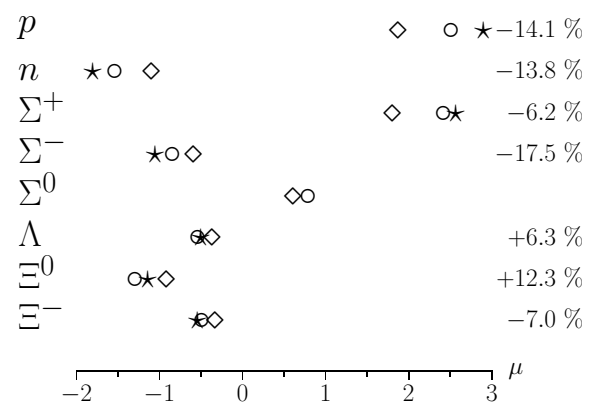

Figure 2. Octet magnetic moments. See text for conventions.

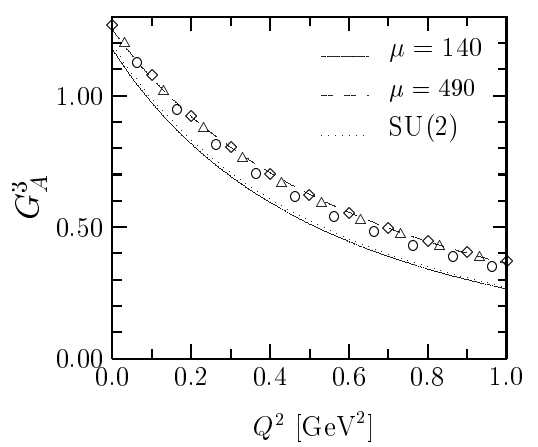

Figure 3. Axial form factor of the nucleon. $\mu$ indicates the meson asymptotics.

mean that the flavor form factor was computed with a profile function which had an asymptotic behaviour determined by the pion $(\mu=140 \mathrm{MeV})$ or kaon $(\mu=490 \mathrm{MeV})$ mass, respectively. The different profile functions are obtained by symmetrically adding contributions to the diagonal matrix element going into the one-particle Dirac Hamiltonian as well as to the nondiagonal mass matrix going into the perturbative mass term. The radii are presented in Tab. 1. The magnetic moments, indicated by $\diamond$ in Fig. 2 and presented in Tab. 1, are given in units of the physical nuclear magneton, while the preferred magnetic moments, denoted by $\circ$ in Fig. 2, are given in units of a magneton defined in terms of the soliton mass ( $\star$ is the experimental value). One of the advantages of Eq. (2) is the improvement in the description of the electric form factor of the neutron, thus indicating that this form factor may be sensitive to the strange content of the nucleon.

The axial form factors are presented in Fig. 3 and Fig. 4 and are obtained in exactly the same framework. Axial constants are presented in Tab. 2. 

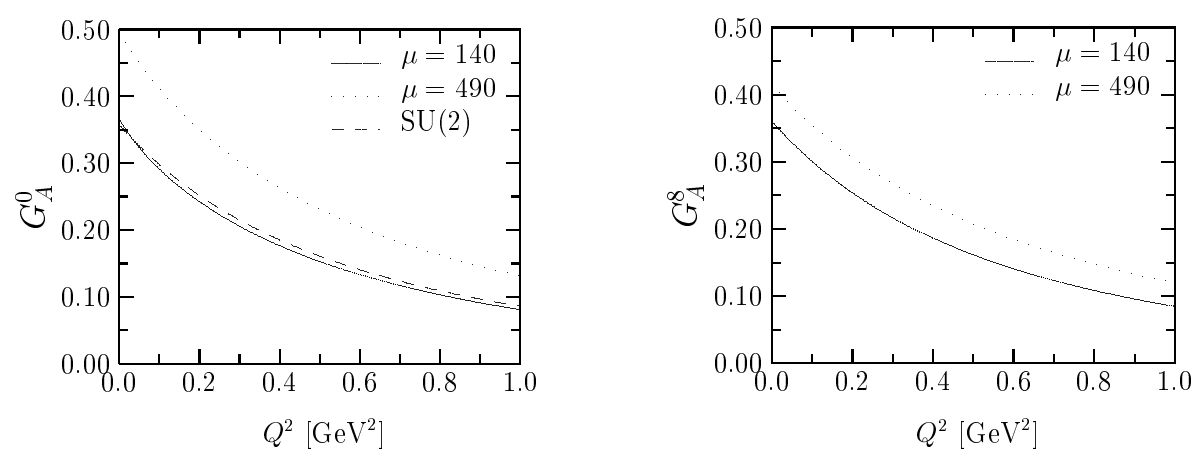

Figure 4. Octet and singlet axial form factors of the nucleon.

Table 2

Axial constants, radii $(\mathrm{fm})$ and dipole masses $\left(\mathrm{GeV} / \mathrm{c}^{2}\right)$.

\begin{tabular}{lcccc}
\hline & SU(2) & \multicolumn{2}{c}{$\mathrm{SU}(3)$} & Exp. \\
\hline$g_{A}^{0}$ & 0.358 & 0.367 & 0.497 & \\
$\left\langle r_{0}^{2}\right\rangle^{1 / 2}$ & 0.662 & 0.844 & 0.703 & \\
$M_{A}^{0}$ & 1.033 & 0.810 & 0.972 & \\
$g_{A}^{3}$ & 1.196 & 1.176 & 1.251 & $1.267 \pm 0.004$ \\
$\left\langle r_{3}^{2}\right\rangle^{1 / 2}$ & 0.666 & 0.732 & 0.568 & $0.635 \pm 0.023$ \\
$M_{A}^{3}$ & 1.026 & 0.934 & 1.202 & $1.077 \pm 0.039$ \\
$g_{A}^{8}$ & & 0.360 & 0.416 & \\
$\left\langle r_{8}^{2}\right\rangle^{1 / 2}$ & & 0.739 & 0.591 & \\
$M_{A}^{8}$ & & 0.926 & 1.156 & \\
\hline$E^{8}$ & & & &
\end{tabular}

Experimental values are from [9].

\section{REFERENCES}

1. C.H. Hyde-Wright and K. de Jager, Ann. Rev. Nuc. Par. Sci. 54 (2004) 217.

2. A. Blotz et al., Nucl. Phys. A 555 (1993) 765.

3. D. Diakonov and V. Y. Petrov, [arXiv:hep-ph/0009006.]

4. C. V. Christov et al., Prog. Part. Nucl. Phys. 37 (1996) 91.

5. M. Praszalowicz, T. Watabe and K. Goeke, Nucl. Phys. A 647 (1999) 49.

6. H. C. Kim, A. Blotz, M. V. Polyakov and K. Goeke, Phys. Rev. D 53 (1996) 4013.

7. I. Eschrich et al. [SELEX Collaboration], Phys. Lett. B 522 (2001) 233.

8. M. I. Adamovich et al. [WA89 Collaboration], Eur. Phys. J. C 8 (1999) 59.

9. A. Liesenfeld et al. [A1 Collaboration], Phys. Lett. B 468 (1999) 20. 